BMJ Open

Diabetes

Research

\& Care

\title{
High-serum carotenoids associated with lower risk for developing type 2 diabetes among Japanese subjects: Mikkabi cohort study
}

\author{
Minoru Sugiura, ${ }^{1}$ Mieko Nakamura, ${ }^{2}$ Kazunori Ogawa, ${ }^{1}$ Yoshinori Ikoma, ${ }^{1}$ \\ Masamichi Yano ${ }^{1}$
}

To cite: Sugiura $M$,

Nakamura M, Ogawa K, et al. High-serum carotenoids associated with lower risk for developing type 2 diabetes among Japanese subjects: Mikkabi cohort study. BMJ Open Diabetes Research and Care 2015;3:e000147. doi:10.1136/bmjdrc-2015000147

Received 5 August 2015 Revised 10 October 2015 Accepted 1 November 2015

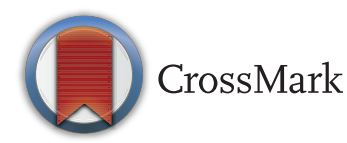

${ }^{1}$ Citrus Research Division, NARO Institute of Fruit Tree Science, National Agriculture and Food Research Organization (NARO), Shizuoka City, Shizuoka, Japan

${ }^{2}$ Department of Community Health and Preventive Medicine, Hamamatsu University School of Medicine, Hamamatsu City, Shizuoka, Japan

Correspondence to Dr Minoru Sugiura; msugiura@affrc.go.jp

\section{ABSTRACT}

Objective: Recent epidemiological studies show the association of antioxidant carotenoids with type 2 diabetes, but thorough longitudinal cohort studies regarding this association have not been well conducted. The objective of this study was to investigate longitudinally whether serum carotenoids are associated with the risk for developing type 2 diabetes among Japanese subjects.

Research design and methods: We conducted a follow-up study on 1073 males and females aged 3079 years at the baseline from the Mikkabi prospective cohort study. Those who participated in the baseline and completed follow-up surveys were examined longitudinally. Over the 10 -year period, 910 subjects (295 males and 615 females) took part in the followup survey at least one time. A cohort of 264 males and 600 females free of diabetes at baseline was studied.

Results: Over a mean follow-up period of 7.8 years ( $S D=2.9), 22$ males and 33 females developed new type 2 diabetes. After adjustments for confounders, the HRs for type 2 diabetes in the highest tertiles of serum $\alpha$-carotene, $\beta$-cryptoxanthin, and total provitamin $\mathrm{A}$ carotenoids against the lowest tertiles were $0.35(95 \%$ $\mathrm{Cl} 0.15$ to 0.82 ), 0.43 (Cl 0.20 to 0.92 ) and 0.41 (Cl 0.19 to 0.90$)$, respectively. For $\beta$-carotene and zeaxanthin, borderline reduced risks were also observed, but these were not significant.

Conclusions: Our results further support the hypothesis that eating a diet rich in carotenoids, especially provitamin A carotenoids, might help prevent the development of type 2 diabetes in Japanese patients.

Trial registration number: NIFT-2013001.

\section{INTRODUCTION}

Type 2 diabetes is the most common chronic disease in the world, and it is emerging as a major medical and public health problem not only in Japan but worldwide. ${ }^{12}$ On the other hand, dietary factors may play an important role in the development of type 2 diabetes. ${ }^{3}$ Antioxidant vitamins and carotenoids are

\section{Key messages}

- Antioxidant micronutrients would be expected to protect against the development of type 2 diabetes, however, evidence from these observational studies is scarce and inconsistent.

- Our results indicated that higher serum $\alpha$-carotene and $\beta$-cryptoxanthin at the baseline were significantly associated with a lower risk of developing type 2 diabetes among Japanese subjects.

- Further evidence from epidemiological research is needed before a definitive conclusion on this issue can be drawn.

\section{Research questions}

- Does antioxidant carotenoid truly help prevent the development of type 2 diabetes?

- Does a diet rich in vegetables and fruits can lower risk of type 2 diabetes?

- Are there race or gender differences about associations of carotenoids and the risk for developing type 2 diabetes?

included in abundance in fruit and vegetables and have been known to contribute to the body's defense against oxidative stress induced by reactive oxygen species and free radicals. ${ }^{45}$ Oxidative stress is thought to play a key role in the pathogenesis of type 2 diabetes by impairing insulin secretion or increasing insulin resistance. ${ }^{6-9}$ Therefore, antioxidant micronutrients, such as vitamins or carotenoids, would be expected to protect against the development of type 2 diabetes. In fact, recent evidence has suggested that antioxidant vitamins and carotenoids may have a protective effect against type 2 diabetes. ${ }^{10-26}$ So far, eight prospective cohort, ${ }^{13-20}$ two nested case-control, ${ }^{21}{ }^{22}$ two casecontrol $^{12} \quad 23$ and three cross-sectional studies ${ }^{24-26}$ have been reported regarding the 
association of serum carotenoids and/or carotenoid intake with the risk for type 2 diabetes and/or high glucose. However, evidence from these observational studies on the associations of carotenoids and the risk for type 2 diabetes is scarce and inconsistent. Furthermore, prospective cohort study concerning the association of serum carotenoids and the risk for developing type 2 diabetes has not been examined among Japanese subjects.

On the other hand, previously, we found that serum antioxidant carotenoids were inversely associated with insulin resistance among middle-aged and older Japanese subjects without diabetes. ${ }^{27}$ In this previous study, we examined the association of the homeostasis model assessment-insulin resistance (HOMA-IR) index with the serum carotenoid concentrations crosssectionally and found that serum lycopene, $\alpha$-carotene, $\beta$-carotene, $\beta$-cryptoxanthin and zeaxanthin were inversely associated with the risk for high HOMA-IR. These results support our hypothesis that antioxidant carotenoids may act as suppressors against the development of insulin resistance and type 2 diabetes. However, this data consisted of cross-sectional analyses. Therefore, only limited inferences can be made regarding temporality and causation. To determine whether antioxidant carotenoids are beneficial micronutrients for preventing type 2 diabetes in Japanese subjects, further cohort studies will be required.

The objective of this study was to investigate longitudinally whether the risk of developing type 2 diabetes is associated with serum carotenoid concentrations in middle-aged and older Japanese subjects. The associations of six serum carotenoid concentrations, that is, lutein, lycopene, $\alpha$-carotene, $\beta$-carotene, $\beta$-cryptoxanthin and zeaxanthin with type 2 diabetes were evaluated longitudinally.

\section{RESEARCH DESIGN AND METHODS Ethics statement}

This study was carried out in accordance with the Declaration of Helsinki and approved by the ethics committee of the NARO Institute of Fruit Tree Science and the Hamamatsu University School of Medicine. We obtained written informed consent from all participants involved in our study.

\section{Study population}

This was a population-based prospective survey involving participants in the Mikkabi Cohort Study conducted in the town of Mikkabi, Shizuoka Prefecture, Japan. The Mikkabi Cohort Study was conducted on two cohorts, one initiated in 2003 (cohort I) and the other in 2005 (cohort II). The study design has been described previously. $^{28}$

After a baseline survey (2003 and 2005), subjects were invited to participate in a follow-up survey in 2005 (for cohort I), 2006, 2007, 2008, 2009 and 2013. Subjects in cohort I were followed from 2005 and subjects in cohort
II from 2006 through September 2013. In this manner, 2-year follow-up data were obtained from 40 participants. In the same way, 3-year, 4-year, 5-year, 6-year, 8-year and 10-year follow-up data were obtained from 41, 95, 47, 161, 94 and 432 participants, respectively. In total, from the six follow-up surveys, 910 subjects (295 males and 615 females) took part in the follow-up survey at least one time. The follow-up rate was $84.9 \%$. The personyears of follow-up were calculated for each subject from the starting point to the date of diagnosis. For this study, we excluded subjects suffering from diabetes (equal to or more than $7 \mathrm{mmol} / \mathrm{L}$ of fasting plasma glucose (FPG)) at the baseline survey as defined by the American Diabetes Association diagnostic criteria. ${ }^{29}$ In addition, those who reported a history of diabetes in the self-administered questionnaire at the baseline survey were excluded. As a result, a total of 264 male and 600 female patients were included in further data analysis.

\section{Blood and anthropometric measurements}

The concentrations of serum carotenoids at the baseline survey, lutein, lycopene, $\alpha$-carotene, $\beta$-carotene, $\beta$-cryptoxanthin and zeaxanthin, were analyzed by reverse-phase high-performance liquid chromatography using $\beta$-apo- $8^{\prime}$-carotenal as an internal standard at the Laboratory of Public Health and Environmental Chemistry, Kyoto Biseibutsu Kenkyusho (Kyoto, Japan), as described previously. ${ }^{30}$ Preceding the study, intraobserver reproducibility of the measurement was evaluated. The range of coefficients of variation of measurements made five times for each of five subjects were 1.9-7.6\% (median, 3\%) for $\alpha$-carotene, $1.1-7 \% \quad(1.2 \%)$ for $\beta$-carotene, $0.9-2.7 \%$ (1.5\%) for $\beta$-cryptoxanthin, $1.6-$ $3.9 \%(3 \%)$ for lutein, $3.4-10.5 \%(6.7 \%)$ for lycopene and $1.7-10.6 \%(3 \%)$ for zeaxanthin. In this study, serum carotenoid concentration was measured by a single technical expert. Quality control of the measurements was assessed at least at 2-3-month intervals using a pooled serum sample. All blood measurements, except for the serum carotenoid concentrations, were conducted at the laboratory of the Seirei Preventive Health Care Center (Shizuoka, Japan). Subjects heights and body weights were measured by trained public health nurses. The body mass index (BMI) was calculated as the body weight $(\mathrm{kg})$ divided by the height $(\mathrm{m})$ squared. Blood pressure was measured using an automated sphygmomanometer, Model BP-103iII (Nihon Colin, Inc, Aichi, Japan).

\section{Self-administered questionnaire}

A self-administered questionnaire was used to collect information about subjects' histories of chronic disease, medication, lifestyle, and dietary intake as described previously. ${ }^{28}$ The assessment of diet was a modification of the validated self-administered 121-item simple foodfrequency questionnaire (FFQ) developed especially for the Japanese. Information about alcohol and the daily intake of nutrients from foods was estimated from 
monthly food intake frequencies using the FFQ analysis software package for Windows (Food Frequency Questionnaire System, System Supply Co, Ltd, Kanagawa, Japan) as described previously. ${ }^{28}$

\section{Ascertainment of type 2 diabetes}

The primary end point was development of type 2 diabetes. Type 2 diabetes was ascertained by the results from a follow-up health examination and selfadministered questionnaire during the 10-year period after the baseline survey. At the six follow-up surveys in 2005 (for cohort I), 2006, 2007, 2008, 2009 and 2013, a diagnosis of diabetes was accepted when any one of the following criteria was met: (1) FPG was equal to or more than $7 \mathrm{mmol} / \mathrm{L}$ or (2) treatment with oral hypoglycemic medication or insulin. Although no specific differentiation between type 1 and type 2 diabetes was made, most cases were apparently type 2 diabetes.

\section{Statistical analysis}

The serum carotenoid concentrations, fasting plasma glucose and serum triacylglycerols were skewed toward higher concentrations. These values were $\log _{\mathrm{e}}$ (natural)transformed to improve the normality of their distribution. An analysis of covariance (ANCOVA) adjusted for age followed by a Bonferroni multiple comparison test was used to compare the means of continuous variables in the three groups stratified by the baseline serum total carotenoid concentration. All variables were presented as an original scale. The data are expressed as the means (SD), geometric mean (95\% CI), or per cent.

To assess the relationship between the serum carotenoid concentrations at the baseline and the development of type 2 diabetes, Cox proportional-hazards regression analyses were performed after excluding patients with a diagnosis of type 2 diabetes at the baseline. Participants were divided into three categories according to tertiles of serum baseline carotenoid concentrations. HRs and 95\% CIs were calculated for the categories of serum carotenoid concentrations at the baseline in tertiles, with the lowest tertiles as the reference, by using the Cox proportional-hazards model and adjusting for potential confounding variables. In the multivariate models, we adjusted each carotenoid concentration into the same model as total carotenoid concentration excluding objective variable. We also assessed linear associations by using the mean values of serum carotenoid concentrations at the baseline for each tertile. All statistical analyses were performed using a statistical software package for Windows (SPSS V.12.0J, SPSS Inc, Chicago, Illinois, USA) on personal computers.

\section{RESULTS}

\section{Baseline characteristics in study subjects}

Table 1 shows the characteristics of study subjects at the baseline survey and the incidence of type 2 diabetes according to tertile of baseline serum total carotenoid concentration. The percentage of male subject and current cigaret smoker, and BMI was significantly low, in accordance with the tertile of baseline serum total carotenoid concentration. Age, FPG, serum total cholesterol, and all six serum carotenoid concentrations at baseline were significantly positively associated with the baseline serum total carotenoid concentration. Although protein, fat, carbohydrate and total energy intakes were not different among three groups, fiber intakes of the middle and highest tertiles were significantly higher compared with that of the lowest group. In contrast, ethanol intakes of the middle and highest tertiles were significantly lower compared with that of the lowest group.

\section{Risk of type 2 diabetes according to tertiles of baseline serum carotenoid concentrations}

The HRs of type 2 diabetes associated with the tertiles of six serum carotenoid concentrations at the baseline survey, after adjusting for confounding factors, are shown in table 2. After adjusting for age, sex and BMI, significantly lower HRs for type 2 diabetes were observed in the highest (T3) groups of serum $\alpha$-carotene, $\beta$-cryptoxanthin and total provitamin A carotenoids ( $\alpha$-carotene and $\beta$-carotene and $\beta$-cryptoxanthin). These significant inverse associations of serum $\alpha$-carotene, $\beta$-cryptoxanthin and total provitamin A carotenoids with the risk for developing type 2 diabetes were also observed after further adjustment for current tobacco use, exercise habits, total energy intake excluding ethanol and ethanol intake. However, these significant associations were not observed after further adjusting for serum total carotenoid concentration excluding each carotenoid as objective variables. On the other hand, for $\beta$-carotene and zeaxanthin, borderline reduced risks were observed, but these were not significant. Other serum carotenoid concentrations such as lutein, lycopene and total serum carotenoid concentration also showed a tendency to inversely associate with the risk for developing type 2 diabetes, but these were not significant. We also examined the association of basal serum carotenoid concentrations with the risk for developing type 2 diabetes among subjects with normal fasting glucose (less than $6.1 \mathrm{mmol} / \mathrm{L}$ of FPG). In the results, significant inverse associations of serum $\alpha$-carotene, $\beta$-cryptoxanthin and total provitamin A carotenoids with the risk for developing type 2 diabetes were also observed after excluding patients with impaired fasting glucose (6.1-6.9 mmol/L of FPG; data not shown).

\section{DISCUSSION}

The objective of this study was to investigate longitudinally whether the incidence of risk for type 2 diabetes is associated with serum carotenoid concentrations in middle-aged and older Japanese subjects. The results indicated that higher serum $\alpha$-carotene and $\beta$-cryptoxanthin at the baseline were significantly associated with a lower risk of developing type 2 diabetes. 
Table 1 Characteristics of the study subject at baseline survey according to tertile of baseline serum total carotenoid concentration*

\begin{tabular}{|c|c|c|c|}
\hline & \multicolumn{3}{|c|}{ Tertiles of baseline serum total carotenoid concentration } \\
\hline & Lowest & Middle & Highest \\
\hline $\mathrm{n}$ & 288 & 288 & 288 \\
\hline Male (\%) & 44.8 & 29.5 & 17.4 \\
\hline Age (years) & $50.4(10.3)$ & $55.9(9.8) \S \S$ & $58.1(8.1) \S \S$ \\
\hline Body mass index $\left(\mathrm{kg} / \mathrm{m}^{2}\right)$ & $23.0(3.4)$ & $22.9(3.1)$ & $22.4(2.7) \S$ \\
\hline Fasting plasma glucose (mmol/L) $\dagger$ & $5.09(5.04$ to 5.15$)$ & $5.17(5.11$ to 5.23$)$ & $5.20(5.14$ to 5.26$) \S$ \\
\hline Systolic blood pressure (mmHg) & $127.5(19.0)$ & $130.8(19.8)$ & $130.3(18.1)$ \\
\hline Serum total cholesterol (mmol/L) & $5.13(0.87)$ & $5.54(0.86) \S \S$ & $5.84(0.79) \S \S$ \\
\hline Serum triacylglycerol $(\mathrm{mmol} / \mathrm{L}) \dagger$ & $1.03(0.97$ to 1.10$)$ & $1.02(0.96$ to 1.08$)$ & 0.98 (0.92 to 1.03$)$ \\
\hline \multicolumn{4}{|l|}{ Dietary intake } \\
\hline \multicolumn{4}{|l|}{ Total energy (kcal/day) } \\
\hline Including ethanol & $2110.4(561.4)$ & $2032.1(506.4)$ & $2022.7(553.4)$ \\
\hline Excluding ethanol & $2001.3(531.6)$ & $1986.6(497.6)$ & $2000.1(546.4)$ \\
\hline Ethanol (g/day) & $16.5(29.6)$ & $6.8(15.2) \S \S$ & $3.1(9.0) \S \S$ \\
\hline Protein (g/day) & $72.7(21.9)$ & $74.4(22.4)$ & $74.6(24.8)$ \\
\hline Fat (g/day) & $55.1(21.2)$ & $53.6(20.2)$ & $53.3(21.8)$ \\
\hline Carbohydrate (g/day) & $300.3(75.7)$ & $295.2(68.7)$ & $298.6(71.8)$ \\
\hline Fiber (g/day) & $11.9(4.9)$ & $13.4(4.6) \S \S$ & $14.8(5.6) \S \S$ \\
\hline \multicolumn{4}{|c|}{ Serum carotenoid concentrations $(\mathrm{mmol} / \mathrm{L}) \dagger$} \\
\hline Lutein & $0.45(0.44$ to 0.47$)$ & $0.58(0.56$ to 0.61$) \S \S$ & $0.67(0.64$ to 0.70$) \S \S$ \\
\hline Lycopene & $0.22(0.20$ to 0.24$)$ & $0.29(0.27$ to 0.31$) \S \S$ & $0.36(0.33$ to 0.39$) \S \S$ \\
\hline$\alpha$-Carotene & 0.09 (0.09 to 0.10$)$ & $0.14(0.13$ to 0.15$) \S \S$ & $0.18(0.17$ to 0.19$) \S \S$ \\
\hline$\beta$-Carotene & $0.36(0.34$ to 0.38$)$ & $0.64(0.61$ to 0.66$) \S \S$ & $1.08(1.03$ to 1.13$) \S \S$ \\
\hline$\beta$-Cryptoxanthn & $0.54(0.51$ to 0.58$)$ & 1.35 (1.28 to 1.42$) \S \S$ & 3.25 (3.10 to 3.42$) \S \S$ \\
\hline Zeaxanthin & $0.19(0.19$ to 0.20$)$ & $0.23(0.22$ to 0.24$) \S \S$ & $0.28(0.27$ to 0.28$) \S \S$ \\
\hline Provitamin A carotenoid & 1.05 (1.00 to 1.10$)$ & 2.25 (2.19 to 2.32$) \S \S$ & 4.69 (4.52 to 4.86$) \S \S$ \\
\hline Non-provitamin A carotenoid & 0.91 (0.88 to 0.94$)$ & $1.16(1.12$ to 1.20$) \S \S$ & $1.36(1.31$ to 1.41$) \S \S$ \\
\hline Total carotenoid & 2.01 (1.95 to 2.08$)$ & 3.50 (3.44 to 3.55$) \S \S$ & 6.16 (5.98 to 6.34$) \S \S$ \\
\hline Current tobacco use (\%) & 20.8 & 9.0 & 2.8 \\
\hline Exercise habits (\%) & 19.9 & 20.9 & 24.5 \\
\hline
\end{tabular}

${ }^{*}$ Data are mean (SD), geometric mean $(95 \% \mathrm{Cl})$, or per cent.

†These variables were represented as original scale after analysis by log (natural) transformed values.

${ }^{\S} p<0.05,{ }^{\S} \mathrm{p}<0.001$ versus the lowest tertile of serum total carotenoid concentration by analysis of covariance (ANCOVA) adjusted for age followed by Bonferroni multiple comparison test.

This is the first cohort study to examine the association of serum carotenoid concentrations with the risk of developing type 2 diabetes among Japanese subjects. Numerous antioxidant vitamins and carotenoids are contained in fruits and vegetables, and several recent epidemiological studies have shown inverse associations of antioxidant vitamin and carotenoid intake or serum levels with type 2 diabetes and/or high glucose. ${ }^{10-27}$ Our results further support the hypothesis that eating a diet rich in carotenoids, especially $\alpha$-carotene and $\beta$-cryptoxanthin, might help prevent the development of type 2 diabetes among middle-aged and older Japanese subjects.

Recent eight prospective cohort have been reported about the association of serum and/or carotenoid intake with the risk for type 2 diabetes and/or high glucose. ${ }^{13-20}$ However, evidence from these observational studies on the associations of carotenoids and the risk for type 2 diabetes is scarce and inconsistent. Six cohort studies have been reported regarding the association of serum and/or carotenoid intake with the risk for type 2 diabetes and/or high glucose, ${ }^{13-15} 17-19$ and two cohort studies show no significant association. ${ }^{16}{ }^{20}$ Kataja-Tuomola et $a l^{16}$ have reported that dietary antioxidants such as tocopherols, vitamin $\mathrm{G}$ and carotenoids were not associated with a decreased risk of incident diabetes in middle-aged male smokers. On the other hand, Hozawa et $a l^{19}$ have found that higher serum carotenoid concentrations are associated with a lower risk of diabetes and insulin resistance in non-smokers but not in smokers. It has been reported that cigaret smoking reduces serum carotenoid concentrations. $^{28} 3132$ Oxidative stress induced by cigaret smoking may interact with the association of serum carotenoids with the risk for developing type 2 diabetes. Additionally, in our study, significant inverse associations of basal serum $\alpha$-carotene and $\beta$-cryptoxanthin with the risk for developing type 2 diabetes were observed among non-smokers (data not shown). However, in the current smokers group, since coefficients did not converge, no further models could be fitted because the sample size of current smokers 
Table 2 HRs (and $95 \% \mathrm{Cls}$ ) of tertiles of baseline serum carotenoid concentration on incidence of type 2 diabetes among total patients

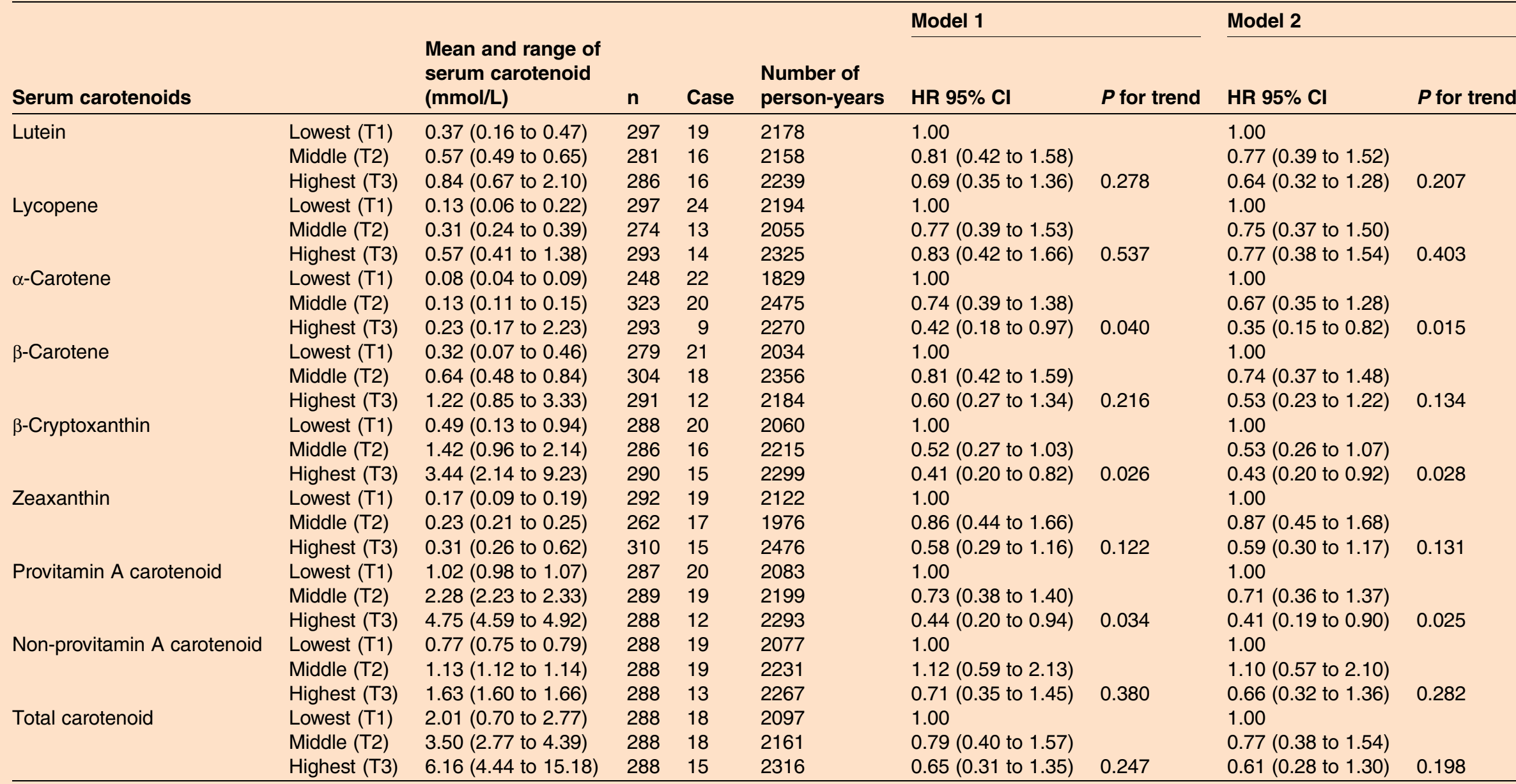

Model 1: Age, sex and BMI were adjusted. Model 2: Current tobacco use, exercise habits, total energy intake excluding ethanol, and ethanol intake were further adjusted.

BMI, body mass index. 
was too small for further data analysis. Another experiment shows that dietary lycopene did not associate with the risk of type 2 diabetes, ${ }^{20}$ and we also found that the basal serum lycopene concentration showed a tendency to inversely associate with the risk of type 2 diabetes, but this was not significant.

On the other hand, four recent large intervention studies show that $\beta$-carotene supplementation had no effect on the incidence of type 2 diabetes and/or fasting plasma glucose. ${ }^{1433-35}$ However, interestingly, Czernichow et $a^{14}$ reported that antioxidant supplementation for 7.5 years did not affect fasting plasma glucose, but baseline $\beta$-carotene dietary intake and serum concentration were inversely associated with FPG in multivariate mixed models using the French randomized placebo-controlled SU.VI.MAX (Supplementation en Vitamines et Mineraux Antioxydants) trial. These results indicate that eating a diet rich in carotenoids may have a protective effect against the development of type 2 diabetes.

In our survey, basal serum carotenoid concentrations, especially $\alpha$-carotene and $\beta$-cryptoxanthin, were inversely associated with the risk for developing type 2 diabetes. $\beta$-Cryptoxanthin is a carotenoid pigment that is particularly abundant in the Japanese mandarin orange. ${ }^{36} 37$ Previously, we found that the serum $\beta$-cryptoxanthin level increases extremely according to an increase of Japanese mandarin intake. ${ }^{38}$ Our Mikkabi cohort study was conducted in the town of Mikkabi, Shizuoka Prefecture, Japan. Mikkabi is located in western Shizuoka, and approximately $40 \%$ of its residents work in agriculture. Fruit trees are the key industry in Mikkabi, which is an important producer of Japanese mandarin oranges in Japan. The subjects in this survey were residents of an area in which the Japanese mandarin orange is considerably more popular than in the rest of Japan. The average amount of fruit intake in the group with the highest serum $\beta$-cryptoxanthin was about $246 \mathrm{~g} /$ day and was approximately equal to three or four pieces of Japanese mandarin orange. Therefore, the serum concentrations of $\beta$-cryptoxanthin in our study population were widely distributed. Interestingly, Montonen $e t a l^{13}$ found that, among six main carotenoids, only $\beta$-cryptoxanthin intake was significantly associated with a reduced risk of type 2 diabetes. Furthermore, recently, $\mathrm{Ni}$ et $a \hat{l}^{39}$ have found that $\beta$-cryptoxanthin administration attenuated insulin resistance and excessive hepatic lipid accumulation and peroxidation, with increases in M1-type macrophages/ Kupffer cells and activated stellate cells, and fibrosis in high-cholesterol and high-fat diet fed mice. Based on these findings, $\beta$-cryptoxanthin might be a useful dietary antioxidant to prevent type 2 diabetes and metabolic disorders. In contrast, $\alpha$-carotene is also widely contained in green and yellow vegetables and fruits. ${ }^{37}$ The serum $\alpha$-carotene concentration in our study population was extremely low compared with the concentration of $\beta$-cryptoxanthin (table 1 ), but basal serum $\alpha$-carotene was significantly associated with a lower risk for developing type 2 diabetes (table 2). We have no clear explanation, as this was observed by chance. We concluded that antioxidant carotenoids might be effective in the prevention of insulin resistance and type 2 diabetes because all six carotenoids showed a tendency to inversely associate with the risk for developing type 2 diabetes in our survey. $\alpha$-Carotene and $\beta$-carotene and $\beta$-cryptoxanthin are provitamin A carotenoids, which will be converted in the body to retinol. Retinoic acid is synthesized intracellularly from retinol and plays a regulatory role in lipid/ glucose homeostasis and type 2 diabetes. ${ }^{40}$ Among six main carotenoids, provitamin A carotenoids might be more effective substances against type 2 diabetes than other non-provitamin A carotenoids, such as lycopene, lutein and zeaxanthin.

On the other hand, it is well known that cigaret smoking and/or alcohol drinking reduces serum carotenoid concentrations. ${ }^{31} 32$ In fact, previously we have also found that cigaret smoking and alcohol drinking may reduce the serum $\beta$-carotene, $\alpha$-carotene and $\beta$-cryptoxanthin concentrations in a synergistic manner. ${ }^{28}$ Furthermore, recently Hozawa $e t a l^{19}$ have found that higher serum carotenoid concentrations are associated with lower risk of diabetes and insulin resistance in nonsmokers but not in smokers. Therefore, we re-examined the associations of basal serum carotenoid concentrations and the risk for developing type 2 diabetes stratified by smoking status and/or alcohol drinking. As results, a significant reduced risk was observed in the highest tertile of serum $\alpha$-carotene among non-smokers $(\mathrm{HR}=0.41,95 \%$ CI 0.18 to 0.93 ). Basal serum $\beta$-cryptoxanthin was also inversely associated with the risk for developing type 2 diabetes, but this was not significance (data not shown). On the other hand, previously we have found that more than $25 \mathrm{~g}$ of daily alcohol intake may reduce the serum $\beta$-carotene, $\alpha$-carotene and $\beta$-cryptoxanthin concentrations. ${ }^{28}$ Therefore, we re-examined the associations of basal serum carotenoid concentrations and the risk for developing type 2 diabetes stratified by alcohol drinking status. As results, significant reduced risks were observed in the highest tertiles of serum $\alpha$-carotene and $\beta$-cryptoxanthin among non-drinkers and light-drinkers (less than $25 \mathrm{~g}$ daily) ( $\mathrm{HR}=0.37,95 \%$ CI 0.17 to 0.83 and $\mathrm{HR}=0.43,95 \%$ CI 0.20 to 0.96 , respectively). In contrast, in the current smokers and/or moderate and heavydrinkers (more than $25 \mathrm{~g}$ daily), since coefficients did not converge, no further models would be fitted, because sample size of current smokers and/or moderate and heavy-drinkers was too small for further data analyses (data not shown). From our results and previous findings, we concluded that carotenoids might help prevent the development of type 2 diabetes in non-smokers and/or non-drinkers and light drinkers rather than current smokers and/or moderate and heavy-drinkers.

This study had some limitations. First, we could not evaluate the association of blood levels of other antioxidants such as vitamins $\mathrm{C}$ and $\mathrm{E}$ with the risk for 
developing type 2 diabetes. It would be necessary to measure the blood levels of vitamins $\mathrm{C}$ and $\mathrm{E}$ in order to examine the association of these antioxidant vitamin concentrations with type 2 diabetes. Second, since we used a single measurement of serum carotenoid concentrations at the baseline, dietary changes of patients were not considered during the follow-up survey. Misclassification of serum carotenoid concentrations relative to long-term average levels was expected. Finally, in our study, the sample size was not particularly large and, thus, had less statistical power. Further studies on a large scale will be required.

In conclusion, this longitudinal cohort study among middle-aged and older Japanese subjects showed that the risk of developing type 2 diabetes was inversely associated with the baseline serum $\alpha$-carotene and $\beta$-cryptoxanthin concentration. Our findings further support the hypothesis that eating a diet rich in antioxidant carotenoids, especially provitamin A carotenoids, might help prevent the development of type 2 diabetes. However, further evidence from epidemiological research is needed before a definitive conclusion on this issue can be drawn.

Acknowledgements The authors are grateful to the participants in our survey and to the staff of the health examination programme for residents of the town of Mikkabi, Shizuoka, Japan. The authors are also grateful to the staff of the Seirei Preventive Health Care Center (Shizuoka, Japan).

Contributors MS was responsible for study design, data collection and data management and carried out the data analysis and wrote the manuscript. MN was responsible for study design, data collection and data management and assisted in manuscript preparation. $\mathrm{KO}, \mathrm{YI}$ and $\mathrm{MY}$ were involved in the data collection and assisted in manuscript preparation.

Funding This work was supported by a grant from the Ministry of Agriculture, Forestry and Fisheries (MAFF) for a food research project titled "Research Project on the Development of Agricultural Products and Foods with Health-Promoting Benefits (NAR0)" (2013-A-10) and a grant from the Council for the Advancement of Fruit Tree Science (2013-001).

\section{Competing interests None declared.}

Patient consent Obtained.

Ethics approval Ethics committee of the NARO Institute of Fruit Tree Science and the Hamamatsu University School of Medicine.

Provenance and peer review Not commissioned; externally peer reviewed.

Data sharing statement No additional data are available.

Open Access This is an Open Access article distributed in accordance with the Creative Commons Attribution Non Commercial (CC BY-NC 4.0) license, which permits others to distribute, remix, adapt, build upon this work noncommercially, and license their derivative works on different terms, provided the original work is properly cited and the use is non-commercial. See: http:// creativecommons.org/licenses/by-nc/4.0/

\section{REFERENCES}

1. Ministry of Health, Labor and Welfare. National Health and Nutrition survey in 2012. http://www.mhlw.go.jp/bunya/kenkou/eiyou/dl/ h24-houkoku.pdf (accessed Apr 2015).

2. Global status report on noncommunicable diseases 2014. Geneva: World Health Organization, 2012.

3. Steyn NP, Mann J, Bennett PH, et al. Diet, nutrition and the prevention of type 2 diabetes. Public Health Nutr 2004;7:147-65.

4. Gutteridge JM. Biological origin of free radicals, and mechanisms of antioxidant protection. Chem Biol Interact 1994;91:133-40.
5. Rock CL, Jacob RA, Bowen PE. Update on the biological characteristics of the antioxidant micronutrients: vitamin $\mathrm{C}$, vitamin $\mathrm{E}$, and the carotenoids. J Am Diet Assoc 1996;96:693-702.

6. Oberley LW. Free radicals and diabetes. Free Radic Biol Med 1988:5:113-24.

7. Dandona $\mathrm{P}$, Thusu $\mathrm{K}$, Cook $\mathrm{S}$, et al. Oxidative damage to DNA in diabetes mellitus. Lancet 1996;347:444-5.

8. Giugliano D, Ceriello A, Paolisso G. Oxidative stress and diabetic vascular complications. Diabetes Care 1996;19:257-67.

9. Hunt JV, Dean RT, Wolff SP. Hydroxyl radical production and autoxidative glycosylation. Glucose autoxidation as the cause of protein damage in the experimental glycation model of diabetes mellitus and ageing. Biochem J 1988;256:205-12.

10. Sinclair AJ, Taylor PB, Lunec J, et al. Low plasma ascorbate levels in patients with type 2 diabetes mellitus consuming adequate dietary vitamin C. Diabet Med 1994;11:893-8.

11. Will JC, Ford ES, Bowman BA. Serum vitamin C concentrations and diabetes: findings from the Third National Health and Nutrition Examination Survey, 1988-1994. Am J Clin Nutr 1999;70:49-52.

12. Abahusain MA, Wright J, Dickerson JW, et al. Retinol, alpha-tocopherol and carotenoids in diabetes. Eur J Clin Nutr 1999;53:630-5.

13. Montonen J, Knekt P, Jarvinen R, et al. Dietary antioxidant intake and risk of type 2 diabetes. Diabetes Care 2004;27:362-6.

14. Czernichow S, Couthouis A, Bertrais S, et al. Antioxidant supplementation does not affect fasting plasma glucose in the Supplementation with Antioxidant Vitamins and Minerals (SU.VI. MAX) study in France: association with dietary intake and plasma concentrations. Am J Clin Nutr 2006;84:395-9.

15. Sluijs I, Cadier E, Beulens JW, et al. Dietary intake of carotenoids and risk of type 2 diabetes. Nutr Metab Cardiovasc Dis 2015;25:376-81.

16. Kataja-Tuomola MK, Kontto JP, Männistö S, et al. Intake of antioxidants and risk of type 2 diabetes in a cohort of male smokers. Eur J Clin Nutr 2011;65:590-7.

17. Arnlöv J, Zethelius B, Risérus U, et al. Serum and dietary beta-carotene and alpha-tocopherol and incidence of type 2 diabetes mellitus in a community-based study of Swedish men: report from the Uppsala Longitudinal Study of Adult Men (ULSAM) study. Diabetologia 2009;52:97-105.

18. Akbaraly TN, Fontbonne A, Favier A, et al. Plasma carotenoids and onset of dysglycemia in an elderly population: results of the Epidemiology of Vascular Ageing Study. Diabetes Care 2008;31:1355-9.

19. Hozawa A, Jacobs DR Jr, Steffes MW, et al. Associations of serum carotenoid concentrations with the development of diabetes and with insulin concentration: interaction with smoking: the Coronary Artery Risk Development in Young Adults (CARDIA) Study. Am J Epidemiol 2006;163:929-37.

20. Wang L, Liu S, Manson JE, et al. The consumption of lycopene and tomato-based food products is not associated with the risk of type 2 diabetes in women. J Nutr 2006;136:620-5.

21. Wang L, Liu S, Pradhan AD, et al. Plasma lycopene, other carotenoids, and the risk of type 2 diabetes in women. $A m \mathrm{~J}$ Epidemiol 2006;164:576-85.

22. Reunanen A, Knekt P, Aaran RK, et al. Serum antioxidants and risk of non-insulin dependent diabetes mellitus. Eur J Clin Nutr 1998;52:89-93.

23. Ford ES, Will JC, Bowman BA, et al. Diabetes mellitus and serum carotenoids: findings from the Third National Health and Nutrition Examination Survey. Am J Epidemiol 1999;149:168-76.

24. Coyne T, Ibiebele TI, Baade PD, et al. Diabetes mellitus and serum carotenoids: findings of a population-based study in Queensland, Australia. Am J Clin Nutr 2005;82:685-93.

25. Ylönen K, Alfthan G, Groop L, et al. Dietary intakes and plasma concentrations of carotenoids and tocopherols in relation to glucose metabolism in subjects at high risk of type 2 diabetes: the Botnia Dietary Study. Am J Clin Nutr 2003;77:1434-41.

26. Suzuki K, Ito $Y$, Nakamura $S$, et al. Relationship between serum carotenoids and hyperglycemia: a population-based cross-sectional study. J Epidemiol 2002;12:357-66.

27. Sugiura M, Nakamura M, Ikoma $Y$, et al. The homeostasis model assessment-insulin resistance index is inversely associated with serum carotenoids in non-diabetic subjects. J Epidemiol 2006;16:71-8.

28. Sugiura M, Nakamura M, Ogawa K, et al. Synergistic interaction of cigarette smoking and alcohol drinking with serum carotenoid concentrations: findings from a middle-aged Japanese population. Br J Nutr 2009;102:1211-19.

29. American Diabetes Association. Diagnosis and classification of diabetes mellitus. Diabetes Care 2010;33(Suppl 1):S62-9.

30. Sugiura $\mathrm{M}$, Nakamura $\mathrm{M}$, Ikoma $\mathrm{Y}$, et al. High serum carotenoids are inversely associated with serum gamma-glutamyltransferase in 
alcohol drinkers within normal liver function. $J$ Epidemiol 2005;15:180-6.

31. Gabriel HE, Liu Z, Crott JW, et al. A comparison of carotenoids, retinoids, and tocopherols in the serum and buccal mucosa of chronic cigarette smokers versus nonsmokers. Cancer Epidemiol Biomarkers Prev 2006;15:993-9.

32. Widome R, Jacobs DR Jr, Hozawa A, et al. Passive smoke exposure and circulating carotenoids in the CARDIA study. Ann Nutr Metab 2010;56:113-18.

33. Liu S, Ajani U, Chae C, et al. Long-term beta-carotene supplementation and risk of type 2 diabetes mellitus: a randomized controlled trial. JAMA 1999;282:1073-5.

34. Kataja-Tuomola M, Sundell JR, Männistö S, et al. Effect of alpha-tocopherol and beta-carotene supplementation on the incidence of type 2 diabetes. Diabetologia 2008;51:47-53.

35. Song Y, Cook NR, Albert CM, et al. Effects of vitamins $C$ and $E$ and beta-carotene on the risk of type 2 diabetes in women at high risk of cardiovascular disease: a randomized controlled trial. Am J Clin Nutr 2009:90:429-37.

36. Goodner KL, Rouseff RL, Hofsommer HJ. Orange, mandarin, and hybrid classification using multivariate statistics based on carotenoid profiles. J Agric Food Chem 2001;49:1146-50.

37. Holden JM, Eldridge AL, Beecher GR, et al. Carotenoid content of U.S. foods: an update of the database. J Food Comp Anal 1999;12:169-96.

38. Sugiura $\mathrm{M}$, Matsumoto $\mathrm{H}$, Kato $\mathrm{M}$, et al. Multiple linear regression analysis of the seasonal changes in the serum concentration of beta-cryptoxanthin. J Nutr Sci Vitaminol 2004;50:196-202.

39. Ni Y, Nagashimada M, Zhan L, et al. Prevention and reversal of lipotoxicity-induced hepatic insulin resistance and steatohepatitis in mice by an antioxidant carotenoid, $\beta$-cryptoxanthin. Endocrinology 2015;156:987-99.

40. Rhee EJ, Plutzky J. Retinoid metabolism and diabetes mellitus. Diabetes Metab J 2012;36:167-80. 